almost negligible.

Some of the bodies showed Alloxane-Schiff and Ninhydrine-Schiff positive reaction. Existence of the nucleic acid in the inclusion body is not to be decisively neglected.

Central part of the inclusion bodies partially does not show metachromasia reaction by toluidine blue staining, and Feulgen's reaction is negative. These central parts seem to have different substance from other part of the inclusion bodies.

As is shown in the table, the outer part of the myoclonus bodies of "Schale" does not show basophil nature, but show positive reaction for PAS, Gomori's silver staining, for pyronine staining and weakly positive reaction for Ninhydrine-Schiff reaction. It seems to consists of different substances from that of the inclusion bodies, and the existence of polysaccharides and protein is not to be neglected.

Above mentioned findings are partialy different from the findings of Har. riman, Seitelberger et al. Perhaps possibility of different histochemical structure of the myoclonus bodies in different cases is not to be denied.

We will continue the study of the myoclonus bodies, the main aspect of which will be the histochemical reaction of nucleic acid, protein and lipid.

We will also proceed the study on the myoclonus epilepsy regarding various organs other than brain and spinal cord.

\title{
A Case of Late Infantile Type of Amaurotic Family Idiocy.
}

\author{
Koshiro Fujrsawa, Toshio Fujisawa \\ Department of Neurology and Psychiatry, Universitry of Tokyo Medical School. \\ Nobuyoshi KawaI. \\ Department of Pathology, University of Tokyo Medical School.
}

Histochemistry of primary cerebral lipidosis is not a completely explored out field of research. Many points of ambiguity and lack of information remain in literatures which cause discrepancy of opinions among authors. We recently experienced one case of late infantile form of amaurotic family idiocy (Bielschowsky), the clinical and autopsy record of which is here by preliminarily reported.

Clinicals : The case is a girl aged 6 years and 10 months old. No pertinent family history except for consanguinity of parental marriage. She has no sibling. She had grown up normal physically and mentally until the onset of present illness at 3 years and 2 months old, when hypersalivation, akinetic- 
astatic seizures and mental retardation began as the preceding symptoms. Loss of vision (opticus atrophy), myoclonus, pyramidal and extrapyramidal signs followed. Continuously progressive, the patient became paretic, bedridden, highly dement, with disturbed consciousness and decerebrate rigidity at the terminal stage. She died from marked general emaciation. Total clinical course extended 3 years and 8 months. Repeated examination of cerebrospinal fluid yielded no pathological findings.

Autopsy : No abnormality in visceral organs. Brain weight $540 \mathrm{~g}$ (half of normal value for the age). Marked symmetrical atrophy of CNS with internal hydrocephalus. Brain was remarkably hard in consistency. Microscopically marked swelling of cell body and processes of nerve cells in whole of grey matter, diffuse fromation of fibre gliosis in whole of white matter and diffuse nerve cell loss of granular layers in both cerebral and cerebellar corticies. No signs of demyelination nor of inflammation were observed.

These findings coincide the histologic picture of amaurotic family idiocy in general. In addition similar type of intracellular deposition of substance was observed in peripheral nerve cells (Auerbach's plexus and sympathetic ganglions) and in histiocytes of spleen.

Histochemistry of storage substance are shown in the Table. Intracellularly stored substances stained strongly by Sudan III, Sudan Black B, PAS and moderately by cupper phthalocyanin in chloroform. Reaction for protein by coupled tetrazonium gives also strongly positive result, while Bial reaction is very ambiguous and questionable. From a series of histochemical procedures the cupper phthalocyanin positive substance is concluded to be sphingomyelin. This finding is contradictory to Diezel's results. One thing notable is, furthermore, distribution of cupper phthalocyanin positive substance in various orgns $\cdots$ it is most prominent in CNS as against lesser in amount in peripheral nerve cells and in histiocytes of spleen. No difference in intracellular content of PAS positive substance was noticed among these organs.

Solubility test by PAS and Sudan Black B stains showed these lipids highly resistant to extraction procedures through various lipid solvents such as pyridin, while exceptionally they dissolved fairly well in $100 \%$ alcohol. Difference of attitude toward PAS stain between nerve cells and microglia cells were found when we examined them on both frozen and paraffin sections. In the former both type of cells stain equally strong with PAS, while in the latter only microglial elements give strong positive reaction. These findings suggest the protein-bound nature of stored lipids and difference of mode of their existence in cytoplasm between in nerve cells and microglial cells.

Stored lipids were eosinophilic, Alcian Blue negative, and orthochromatic in Pfeiffer's and Feyrter's solution, although they stain blue in Nile Blue. Therefore we could not conclude the acidic nature of this glycolipids from these results alone.

In summary we have found hitherto in this case two types of lipids in intracellular storage substance.. glycolipids and sphingomyelin, of which the former excels in amount. Glycolipids are thought to be protein-bound, differ 
in mode of existence in between nerve cells and microglia cells and could not be decided as acidic. Sphingomyelin also exists in considerable amount, although less than glycolipids. Its distribution in various is not uniform ... predominantly found in CNS, in lesser amount inperipheral nervous system and spleen. Ganglioside could not be detected histochemically, as Bial's reaction gave very questionable result.

Table. Histochemistry of Storage Substances in Nerve and Glia Cells

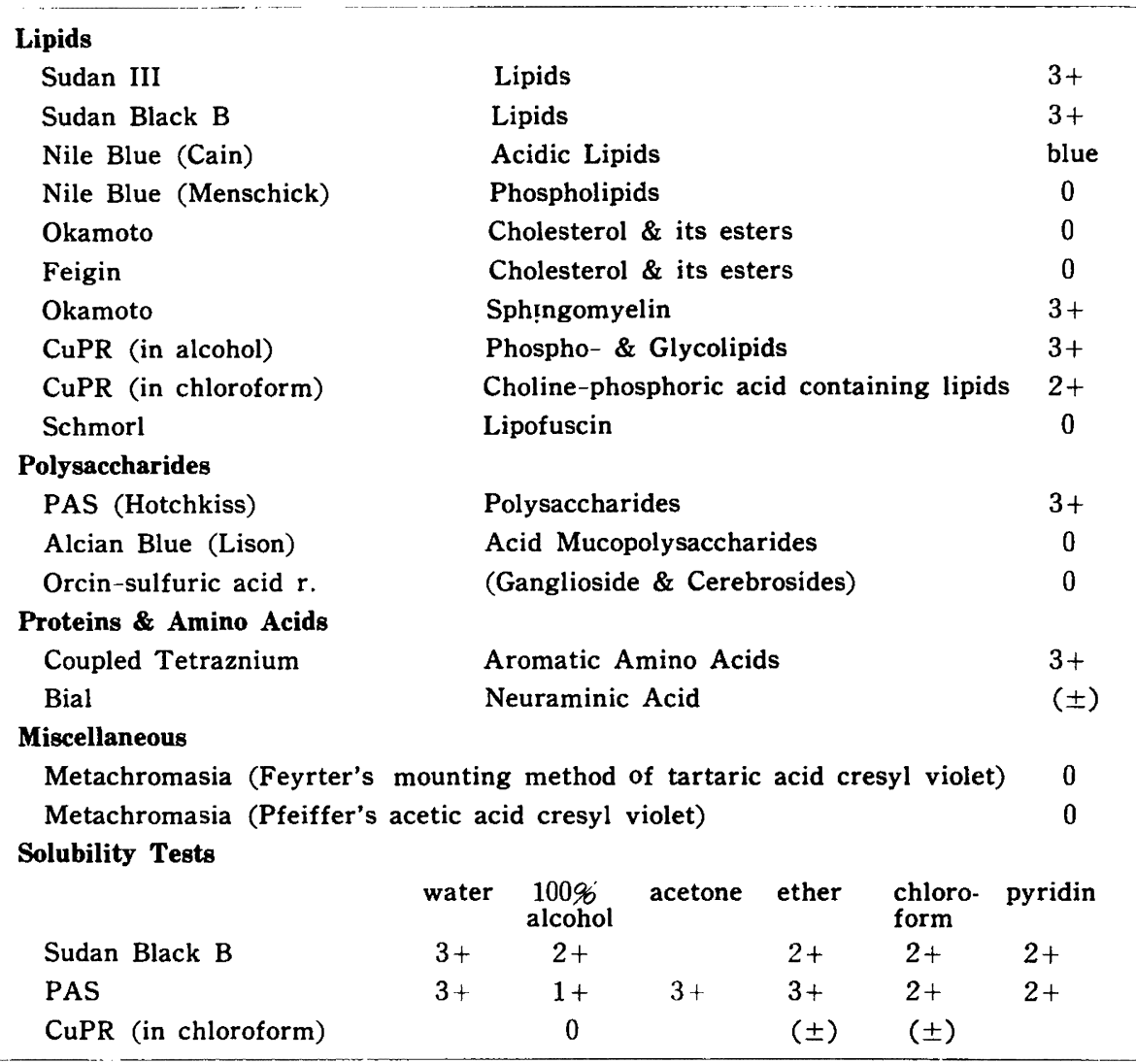

Study of intracellular storage substance by chemical methods and more detailed examination of histological findings are in progress, which will be published $\mathrm{n}$ another paper as a whole. 